\title{
Grounding the Human Body Improves Facial Blood Flow Regulation: Results of a Randomized, Placebo Controlled Pilot Study
}

\author{
Gaétan Chevalier \\ Developmental and Cell Biology Department, University of California at Irvine, Irvine, USA \\ Email: dlbogc@sbcglobal.net
}

Received 27 September 2014; revised 28 October 2014; accepted 3 November 2014

Copyright (C) 2014 by author and Scientific Research Publishing Inc.

This work is licensed under the Creative Commons Attribution International License (CC BY). http://creativecommons.org/licenses/by/4.0/

(c) (i) Open Access

\begin{abstract}
Earthing (grounding) refers to bringing the human body in direct contact with the negative electric charge of the earth's surface by barefoot exposure outdoors or using special conductive indoor systems that are connected to the Earth. To determine if earthing improves facial blood circulation/flow, a double-blind study was designed with forty subjects either grounded or sham-grounded ( 27 grounded subjects and 13 sham-grounded subjects acting as controls) for at least one hour in a comfortable recliner chair equipped with conductive mat, pillow, and patches. The grounding systems were either grounded or sham-grounded via a wire to the ground port (third hole) of a power outlet. A Laser Speckle Contrast Imaging camera was used to continuously record changes in facial blood flow non-invasively. Facial blood flow regulation clearly improved among groundedbut not sham-grounded-subjects. The results demonstrate, for the first time, that even one-hour contact with the earth restores blood flow regulation to the face suggesting enhanced skin tissue repair and improved facial appearance with possible implications for overall health. Further studies, using larger comparison groups, longer monitoring times, and more measuring methods, are warranted in order to confirm the novel influence of the Earth as a protector of skin health and appearance.
\end{abstract}

Keywords

Earthing, Grounding, Laser Speckle Contrast Imaging, Facial Blood Flow

\section{Introduction}

Earthing (or grounding, both words will be used interchangeably) is a practice whereby individuals are put in

How to cite this paper: Chevalier, G. (2014) Grounding the Human Body Improves Facial Blood Flow Regulation: Results of a Randomized, Placebo Controlled Pilot Study. Journal of Cosmetics, Dermatological Sciences and Applications, 4, $293-308$. http://dx.doi.org/10.4236/jcdsa.2014.45039 
direct contact with the surface of the Earth. It includes walking barefoot outdoors, swimming in oceans and lakes, or sleeping, working and relaxing indoors with bare skin in contact with conductive mats, bed sheets, pillows, body bands and patches in order to maintain the body at Earth's electric potential. Unlike past cultures, most people today, particularly in industrial societies, rarely are in contact with the surface of the Earth. They wear shoes with synthetic soles that insulate them from the Earth's electric charge, and they no longer sleep on the ground. The Earth's negative electric surface charge is a virtually limitless reservoir of free electrons that is constantly replenished by the global atmospheric electric circuit [1] [2]. The Earthing hypothesis states that when direct skin contact is made with the Earth's surface or a grounded system indoors, the body's electric potential equalizes with the Earth's potential thereby maintaining the body's access to the Earth's negative surface charge (electrons). This contact with the Earth naturally prevents buildup of static electric charge on the body [3] and allows the body to store a supply of electrons [4] [5].

Published research indicates that Earthing yields a broad array of intriguing positive changes within the physiology and the bioelectrical construct of the body. Multiple reported benefits include improved sleep, decreased pain, a normalizing effect on cortisol, reduction of stress, diminished damage to muscles caused by delayed onset muscle soreness (DOMS), lessening of primary indicators of osteoporosis, and improved thyroid function, glucose regulation, immune response, and blood fluidity. A review of documented benefits of Earthing was published in 2012 [6].

Besides treatments such as plastic surgery (face lift), injections of botox, concentrated platelets and restylane, increasing blood flow to the face is seen as a major natural way to rejuvenate the skin of the face. There are a number of treatments to increase blood flow. Some use creams containing specific ingredients and others use direct skin stimulation (massages, ultrasound and lasers), chemical peel and dermabrasion [7]-[9]. While these treatments give results, they may have important short term or long term side effects; also some of these procedures require intensive post-treatment care and/or prolonged downtime. For example, many procedures designed to induce a controlled form of skin wound to promote dermal matrix remodeling and collagen synthesis require significant post-treatment care and may lead to complications, such as infection, pain, erythema, bleeding, oozing, burns and scaring [9]. Earthing, by comparison, is not a treatment per se. but a simple practice requiring little or no effort, which can be introduced easily into one's daily life.

The present double-blind study was designed to determine if Earthing for one hour produced measurable changes in facial blood flow (FBF). Based on previous studies, the hypothesis is that there will be a marked increase in FBF in grounded subjects vs. ungrounded subjects, as measured by the Laser Speckle Contrast method. Confirmation will suggest that Earthing is an effective and natural way to rejuvenate facial skin and appearance.

\section{Materials and Methods}

This pilot study was approved by BioMed IRB of San Diego, California (http://www.biomedirb.com/) and was conducted at a single center: Total Thermal Imaging, La Mesa, California.

\subsection{Subjects}

Forty (40) participants were recruited with an average age and standard deviation (SD) of $54.8 \pm 9.8$ (details in Table 1). Subjects were randomly assigned to 2 groups: Group A, with 27 grounded individuals; Group B, with 13 sham-grounded individuals (the control group). Subjects were scheduled in the order they signed up to participate.

Exclusion criteria were:

$\diamond$ Pregnancy;

$\diamond$ Below the age of 18 or above 70 ;

$\diamond$ Taking pain, anti-inflammatory medication, sedatives or prescription sleeping medication (less than 3 days prior to testing);

$\diamond$ Taking psychotropic drugs or diagnosed with mental disorder;

$\diamond$ Recent surgery (less than 3 months);

$\diamond$ Documented life threatening disease (such as cancer and AIDS);

$\diamond$ Consumption of alcohol within 48 hours of participation;

$\diamond$ Smoking;

$\diamond$ Use of recreational drugs; 
Table 1. Age and gender distribution of subjects.

\begin{tabular}{ccccc}
\hline & Grounded & \multicolumn{2}{c}{ Controls } \\
\hline & Female & Male & Female & Male \\
\hline No. of Subjects & 20 & 7 & 9 & 4 \\
Average Age & 54.2 & 55.6 & 58.7 & 47.8 \\
Age SD & 10.2 & 12.1 & 6.48 & 8.73 \\
\hline
\end{tabular}

$\diamond$ Previous utilization of Earthing products or similar grounding products;

$\diamond$ Going barefoot outside more than once a week and for more than half hour.

All potential subjects not fitting one or more of the exclusion criteria above were eligible to participate (i.e. there was no specific inclusion criterion).

\subsection{Materials}

Grounding equipment included conductive mats, pillows, and transcutaneous electrical nerve stimulation (TENS) patches (Earthing.com, Palm Springs, California, USA).

\subsection{Earthing (Grounding) Method}

Subjects were grounded with the use of a grounding mat, pillow and conductive patches connected, via conducting wires, into the ground port (third hole) of an electric power outlet. For sham-grounded subjects, conducting wires were similarly connected to the ground port, but modified into an open circuit to block conduction with the Earth's surface. The facility's grounding system was tested and found to be fully functional. All grounding wires contained a built-in $100 \mathrm{k} \Omega$ resistor for surge protection.

\subsection{Measurements and Instrumentation}

Changes in FBF were documented with the Laser Speckle Contrast Imaging (LSCI) technique, also called Laser Speckle Contrast Analysis (LASCA) [10] [11], that delivers real-time, high-resolution blood flow videos (MoorFLPI-2 Speckle Contrast Imager, Moor Instruments Ltd., Axminster, UK; website: http://us.moor.co.uk/ product/ moorflpi-2-speckle-contrast-imager/291).

The LSCI camera illuminates a selected area of tissue with low intensity laser light to produce a high contrast random interference effect known as a speckle pattern. The image processing software uses the fact that high perfusion produces rapid variation in the laser speckle pattern, which is integrated by the charge-coupled device (CCD) camera to produce an area of low contrast (seen as blurring of the speckle pattern in the video image). Conversely, low perfusion causes little variation in the speckle pattern and as a result a high contrast area of well-defined speckle is produced in the video image. Contrast is quantified and the resulting flux is color-coded to produce a perfusion image [10]-[12]. The LSCI camera measures to a maximum skin depth of approximately $1 \mathrm{~mm}$, thus covering mainly superficial skin blood flow [12].

The LSCI camera uses a near-infrared laser diode emitting at a wavelength of $785 \mathrm{~nm}$ and a $568 \times 760$ pixels CCD camera to capture blood flow over an area of up to $80 \times 120 \mathrm{~mm}^{2}$ (the working distance of the camera for providing reliable images is between 15 and $45 \mathrm{~cm}$ ). Researchers and manufacturers generally agree that because of the nature of the flow in capillaries and connecting small blood vessels and the effect of varying skin color and structure, it is not appropriate to use absolute flow units such as $\mathrm{ml} / 100 \mathrm{gm} / \mathrm{minute}$. To justify the use of these units it is necessary to calibrate for the particular tissue type and site of the measurement, which is impractical except in special circumstances and not appropriate for normal day-to-day measurements. Consequently, arbitrary units are used for flux (blood flow) in common with most manufacturers' recommendation [12].

The LSCI camera has the capability of recording up to 25 images per second (called frames per second, or FPS $)$ in standard resolution mode $(152 \times 113$ pixels $)$ and 1 FPS in high resolution mode $(568 \times 760$ pixels $)$ and to put them in a video file. In the present study, an intermediate frame rate of 10 FPS with an averaging period of 10 seconds was used. With this setup, each recorded image represents the average of 100 consecutive frames. 
This setup has the advantage of eliminating very short-term $(<1 \mathrm{sec})$ artifacts while at the same time enhancing durable blood flow features and real FBF changes over time. Blood flow analysis was conducted using appropriate computer software (MoorFLPI Review V4.0, Moor Instruments Ltd., Axminster, UK) installed on a standard desktop computer.

For each image recorded in the video, the analysis software averages the blood flow of the entire face to give a mean FBF value (or flux). The mean FBF values are then processed by the analysis software to generate a graph of mean FBF value over time (with a time increment of 10 seconds between recorded images).

\subsection{Procedure}

Each subject was tested in one individual session. Each grounding or sham-grounding session lasted approximately one hour, during which time the subject sat in a comfortable recliner chair. The reclining angle of the chair was adjusted to a comfortable 30 degrees in respect to the plane of the floor. The chair back and seat were covered with a grounding mat. A grounding pillow was placed at the head position, with a Styrofoam pad positioned under the pillow on each side to help stabilize the head and minimize movements. Patches were placed on both palms and soles (total of four patches). The connector ends of the wires from the patches, pillow, and mat were inserted into the jacks of a connector box placed next to the chair. The box, in turn, was connected by a single wire to the ground port of an adjacent power outlet. To allow or interrupt the conduction, a switch was installed in the middle of that single wire, between the connector box and the ground port. Once the subject was comfortably installed, the camera was positioned and turned on to record the subject's session.

The first ten minutes of each session was dedicated to collecting baseline measurements. After ten minutes, the switch was flipped allowing conduction in the single wire connecting to the ground port. However, the wires used to connect the mat, pillow and patches to the connector box did not permit grounding during the shamgrounding sessions. For both grounded and sham-grounded sessions, the grounding switch was turned off after at least one hour.

A double-blind procedure prevented researchers, study coordinators/technicians, and subjects from knowing whether an individual subject was actually grounded or sham-grounded. To accommodate the double blinded study design calling for about twice as many grounded subjects than sham-grounded subjects, three different colored-coded wires connecting the patches, pillow, and mat to the connector box were utilized. Wires with red and yellow tags permitted grounding; wires with the blue tag did not. The wires' color for a particular session was randomly selected by the study coordinators.

\section{Results}

Varying individual responses of the subjects dictated presenting only individual cases and space constrains to limit results presentation to three grounded subjects (A, B and C) and three sham-grounded (control) subjects $(\mathrm{D}, \mathrm{E}$ and F). The results of these subjects were representative of the specific group results and are presented below according to age (youngest to oldest) for each group.

Linear regression analysis was applied to several graphs when appropriate. Curve smoothing was performed using the central moving average method with 5 points. Both regression analysis and curve smoothing were performed using Microsoft Office Excel 2003 SP2 software.

For each subject, two FBF images are presented first. The first image was extracted from the video just after an initial relaxation period or at 20 minutes after the start of the session when no clear relaxation period could be identified. The relaxation period is the time it takes for FBF to stabilize to a low value at the beginning of a session (corresponding to the time it takes for a subject to relax). The second image was extracted towards the end of the session for the sham-grounded session. After the relaxation period, peaks in blood flow were observed only among grounded subjects. For these subjects, the second image was taken at the highest point of the highest peak after the relaxation period.

Secondly, raw graphs of FBF values over time as calculated by the analysis software are presented. Each time a subject moved the head, a dip in mean FBF value (flux) can be seen, which is a movement artifact not related to the real mean FBF value. Blue arrows were added to these graphs to indicate when such movement artifact occurred.

Thirdly, the raw graphs were corrected for artifacts when needed and smoothed. Each dip in mean FBF value 
was replaced by the average of the mean FBF value immediately before and after the dip and then smoothed according to the procedure already described. Additionally, linear regression analysis was performed when a clear linear tendency could be found.

\subsection{Grounded Subjects}

\subsubsection{Subject A-Female, Caucasian, 33}

Subject A came with an overall body pain level at 3 (on a scale from 1 to 10). Most of the entire neck/back/ arms/ forearms/thighs/legs dropped to a 1 level after one hour of grounding. Figure 1 shows two FBF images as recorded by the camera. The left image was taken at 11 minutes and 50 seconds ( 710 seconds, equivalent to frame 71, with each frame equal to 10 seconds) which corresponds to the end of the relaxation period. The right image was taken at 45 minutes and 10 seconds (2710 seconds, frame 271), corresponding to the highest mean FBF value of the highest peak after relaxation. Each time she moved her head, a dip in blood flow can be seen (movement artifact) in the top graph of Figure 2, which shows unprocessed mean FBF values (flux) over time. These dips are noted using blue arrows. The bottom graph of Figure 2 shows the same graph but corrected for artifacts and smoothed. Each dip was replaced using the method previously described and then smoothed. From Figure 2, it can be seen that mean FBF decreased for about 12 minutes (corresponding to the relaxation period), remained more or less stable for another 28 minutes (with periodic peaks and troughs) and started to increase after that. The peaks and troughs produce a rhythmic fluctuation in mean FBF with a periodicity of 4 minutes and 20 seconds ( 260 seconds, corresponding to 26 periods of 10 seconds, as noted in the figure) that started after the relaxation period.

\subsubsection{Subject B-Female, Caucasian, 49}

Figure 3 shows FBF images of Subject B at 20 minutes (left image), corresponding to the end of the relaxation period, and 39 minutes and 20 seconds (right image), corresponding to the high value of the highest peak after relaxation. Figure 4 shows mean FBF values (flux) over time. In the top graph, which shows unprocessed mean FBF values, only one movement artifact was noted, indicating a very stable position for the entire session. The bottom graph shows mean FBF over time corrected for the artifact and smoothed. After the initial relaxation pe-
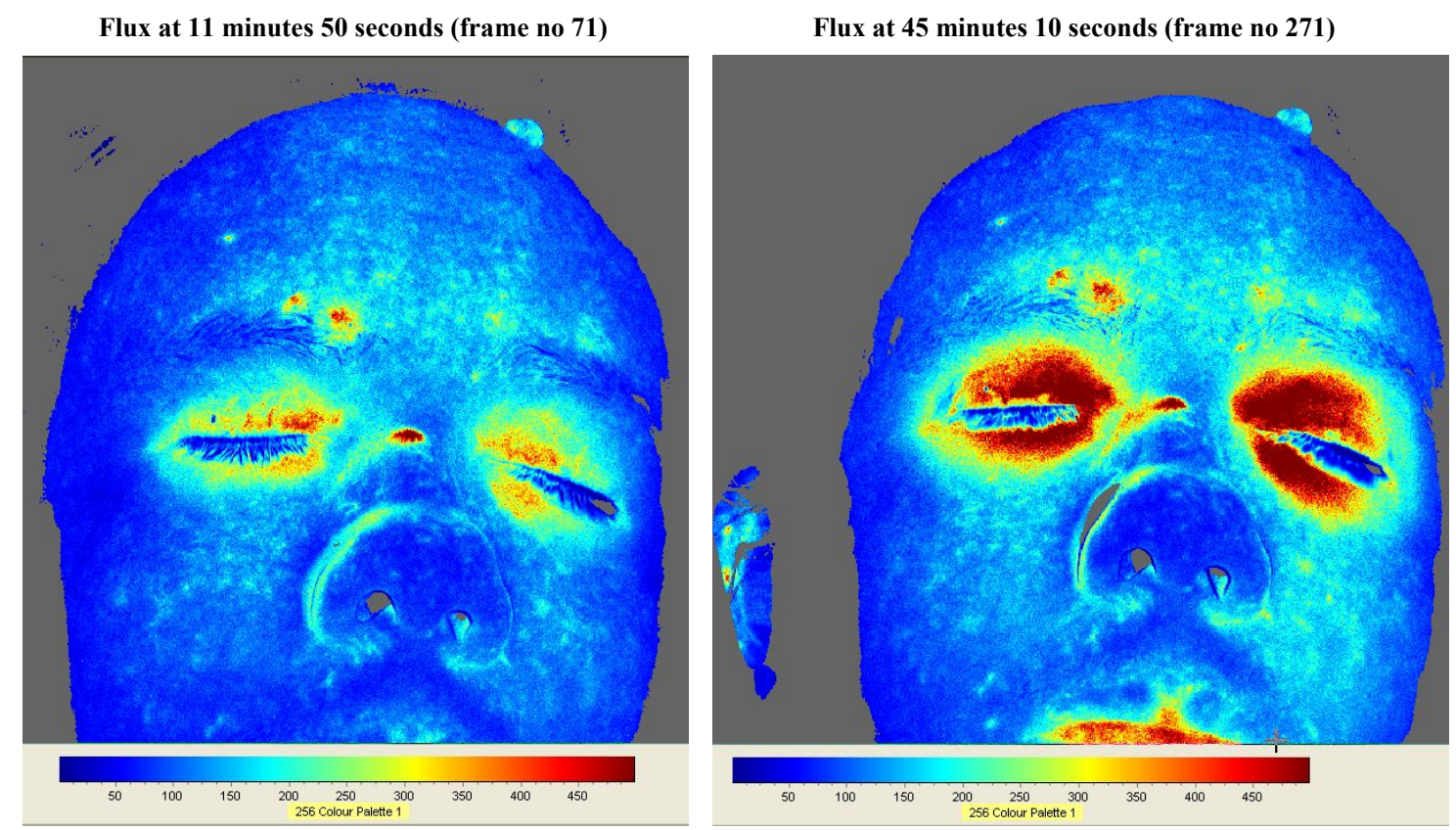

Figure 1. Subject A FBF images at 11 minutes and 50 seconds (left image) and 45 minutes and 10 seconds (right image). The flux intensity scale is shown below each image (dark blue = lowest flux; dark red = highest flux). There is a clear increase in FBF in the right image compared to the left image, especially around the eyes and the cheeks. Descriptive statistics for the left image: Mean Flux $=118.8 ; \mathrm{SD}=59.2$; Flux $\min =0$; Flux $\max =1129$. Descriptive statistics for the right image: Mean Flux $=162.4 ; \mathrm{SD}=105.9$; Flux $\min =0$; Flux $\max =1053$. 

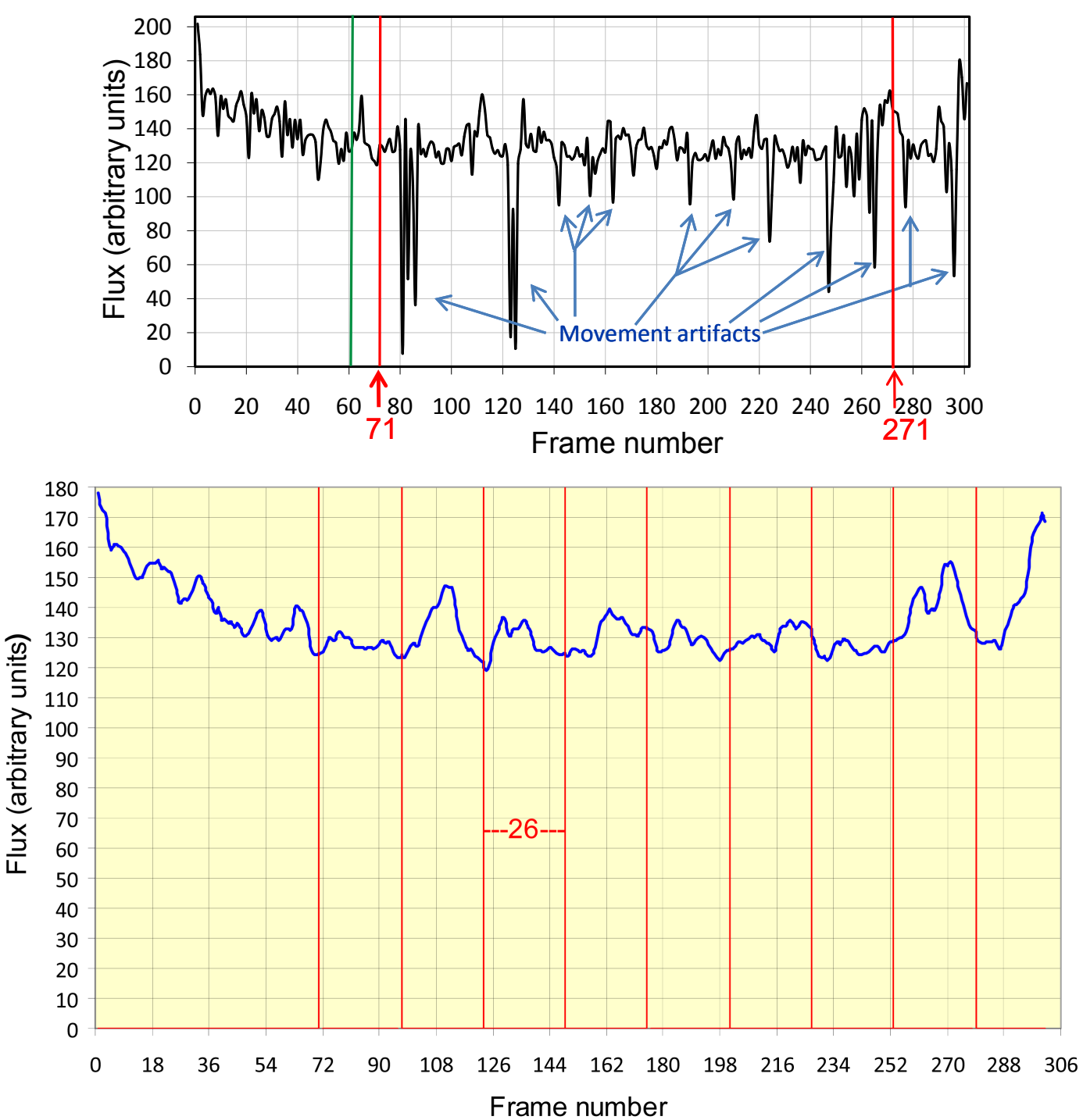

Figure 2. Top graph: Subject A unprocessed graph of mean FBF (in arbitrary units) over time. Red lines and numbers show the times at which the two images of Figure 1 were extracted from the video. Blue arrows point to dips in flux caused by movement artifacts. The green line at 60 shows the time the grounding period started (10 minutes). Bottom graph: Same graph of mean FBF over time corrected for movement artifacts and smoothed. A rhythmic pattern of flux increases and decreases can be observed every 260 seconds (4 minutes and 20 seconds).

riod, no systematic increase in FBF is seen with time, only a rhythmic pattern of means FBF increases and decreases with a periodicity of 16 minutes (960 seconds).

\subsubsection{Subject C-Female, Caucasian, 55}

Figure 5 shows FBF of Subject $C$ at 28 minutes and 40 seconds (left image), corresponding to the end of the relaxation period) and 56 minutes and 40 seconds (right image), corresponding to the high value of the highest peak after relaxation. The top graph of Figure 6 shows unprocessed mean FBF values over time, wherein no movement artifact was noted, indicating very stable head position. The bottom graph of Figure 6 shows the same graph but smoothed (no artifact correction needed). Mean FBF started to increase about 40 minutes in the session. On top of that systematic increase, a rhythmic pattern of mean FBF fluctuations can be seen this time with a periodicity of 530 seconds ( 8 minutes and 50 seconds). It is interesting to note that the rhythmic fluctuations started before the relaxation period was completed. 


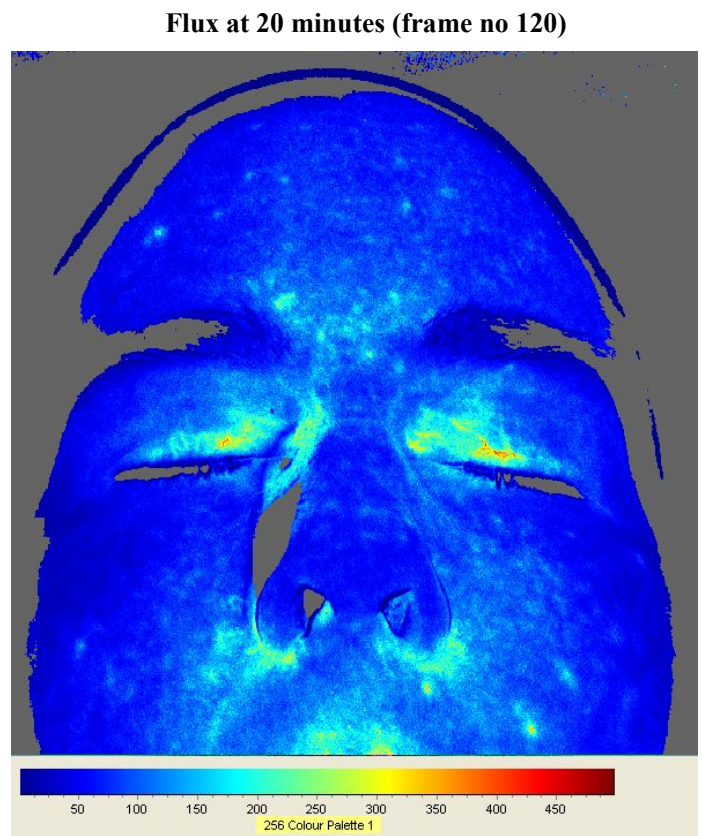

Flux at 39 minutes and 20 seconds (frame no 236)

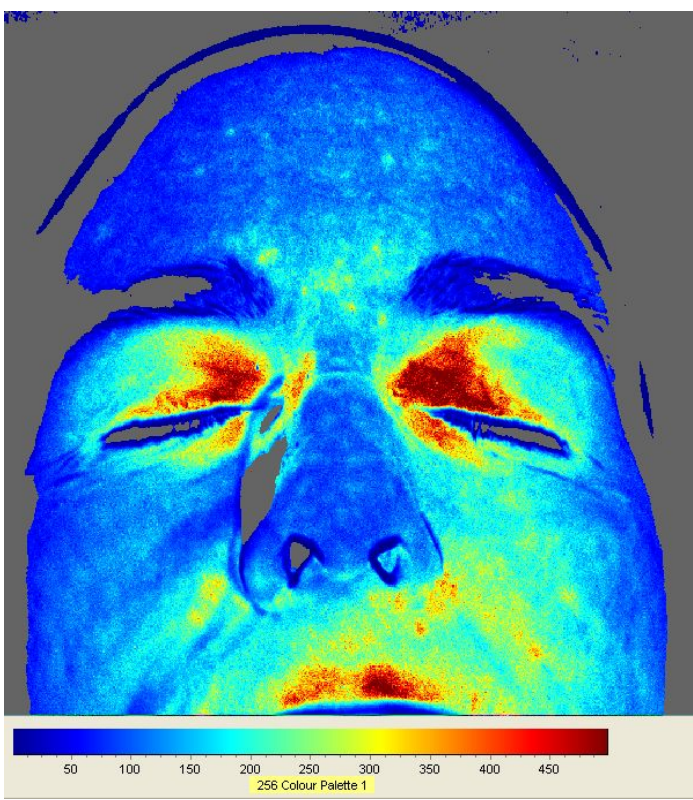

Figure 3. Subject B FBF images at 20 minutes (left image) and 39 minutes and 20 seconds (right image). There is a clear increase in FBF in the right image compared to the left image. Descriptive statistics for the left image: Mean Flux $=91.2 ; \mathrm{SD}=54.3$; Flux $\min =0$; Flux $\max =548$. Descriptive statistics for the right image: Mean Flux $=$ 150.8; $\mathrm{SD}=93.9 ;$ Flux $\min =0 ;$ Flux $\max =810$.
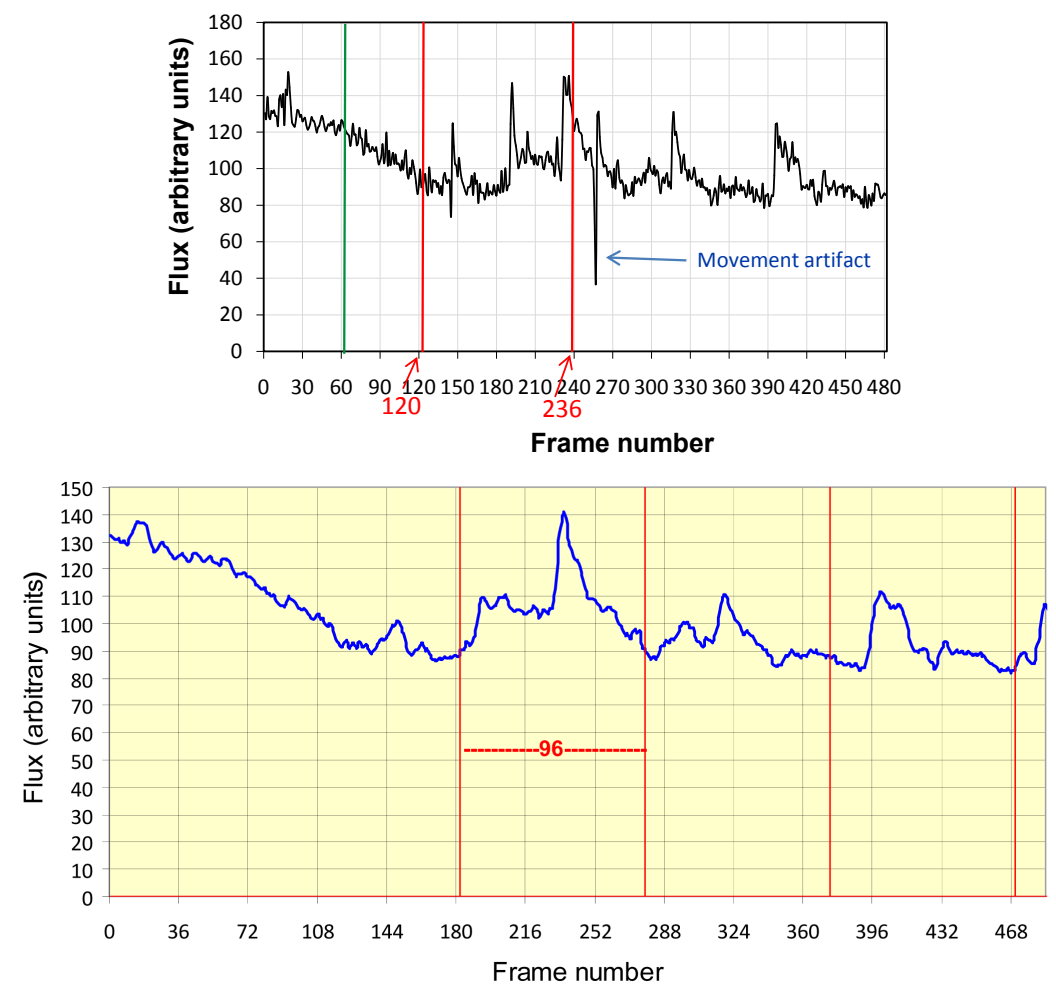

Figure 4. Top graph: Subject B unprocessed graph of mean FBF (in arbitrary units) over time. Red lines and numbers show the times at which the two images of Figure 3 were extracted from the video. The blue arrow points to a dip in flux caused by movement artifacts. The green line at 60 shows the time the grounding period started. Bottom graph: same graph of mean FBF over time corrected for movement artifacts and smoothed. A rhythmic pattern of mean FBF increases and decreases can be observed every 960 seconds (16 minutes). 
Flux at 28 minutes and 40 seconds (frame no 172)

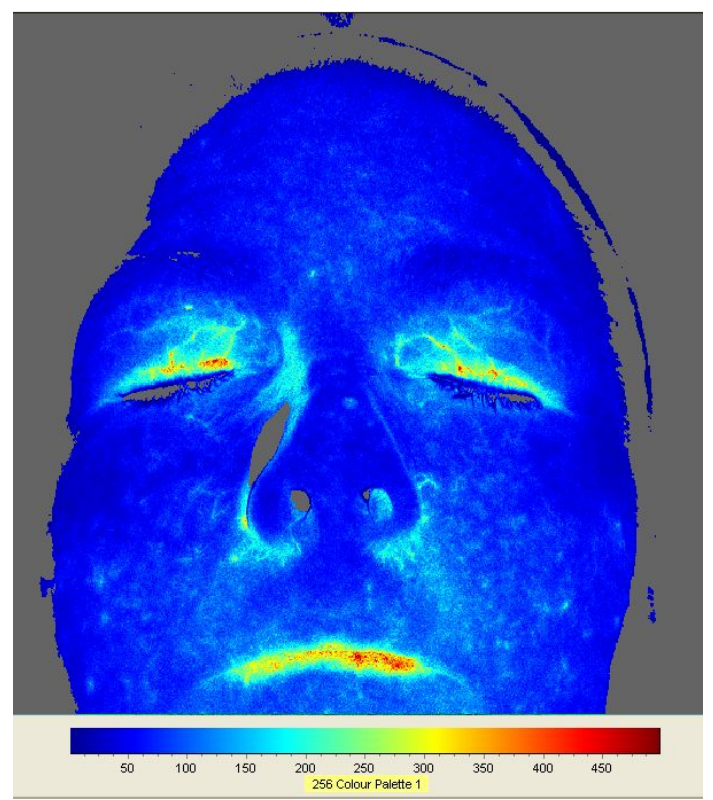

Flux at 56 minutes and 40 seconds (frame no 340)

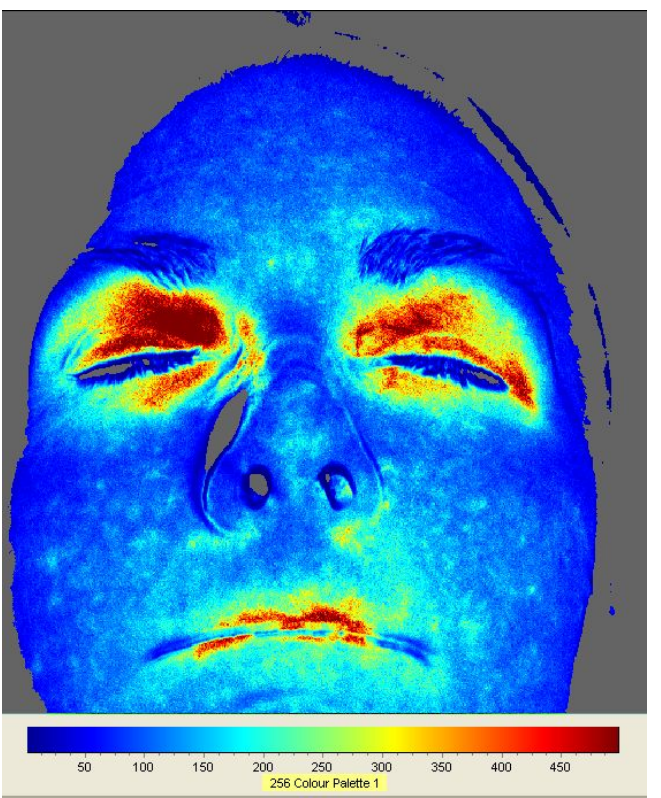

Figure 5. Subject C FBF images at 28 minutes and 40 seconds (left image) and 56 minutes and 40 seconds (right image). There is a clear increase in FBF in the right image compared to the left image. Descriptive statistics for the left image: Mean Flux $=78.5 ; \mathrm{SD}=47.6$; Flux $\min =0$; Flux $\max =517$. Descriptive statistics for the right image: Mean Flux $=141.2 ; \mathrm{SD}=97.6 ;$ Flux $\min =0 ;$ Flux $\max =1050$.
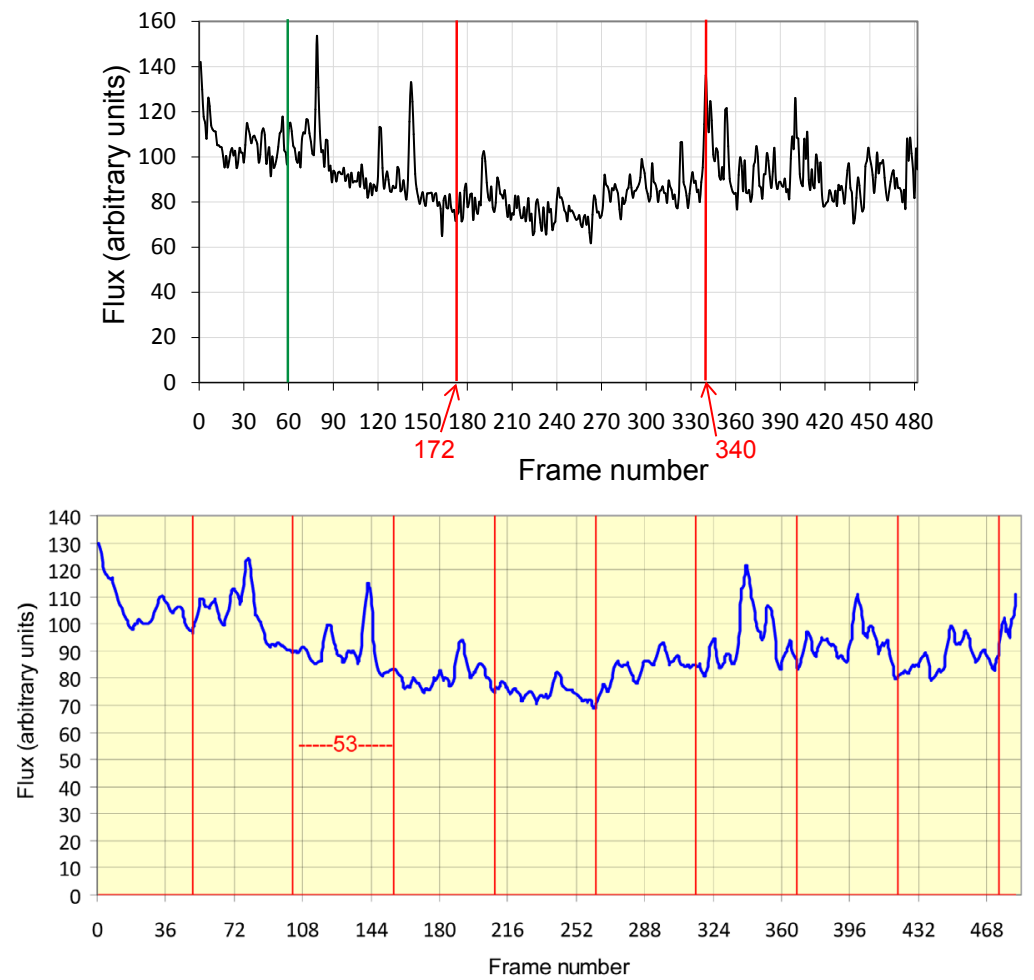

Figure 6. Top graph: Subject C unprocessed graph of mean FBF (in arbitrary units) over time. Red lines and numbers show the times at which the two images of Figure 5 were extracted from the video. The green line at 60 shows the time the grounding period started. Bottom graph: same graph of mean FBF over time corrected for movement artifacts and smoothed. A rhythmic pattern of mean FBF increases and decreases can be observed every 530 seconds ( 8 minutes and 50 seconds). 


\subsection{Sham-Grounded (Control) Subjects}

\subsubsection{Subject D—Male, Caucasian, 42}

Figure 7 shows Subject D FBF images at 20 minutes (left image) and 54 minutes (right image). Figure 8 shows change in mean FBF over time. In the top graph, which presents unprocessed mean FBF values, many movement artifacts are seen as noted. The bottom graph shows top graph data corrected and smoothed for movement artifacts. Linear regression analysis was performed on the bottom graph of Figure 8 and shows that mean FBF decreased linearly with time (coefficient of determination $\mathrm{R}^{2}=0.7171$ ), which explains the lower FBF observed in Figure 7 at 54 minutes. No rhythmic pattern of fluctuations in mean FBF values can be observed.

\subsubsection{Subject E-Female, Caucasian, 55}

Figure 9 shows FBF of subject $\mathrm{E}$ at 20 minutes (left image) and 79 minutes (right image). It is apparent that FBF in this sham-grounded subject is lower at 79 minutes. Figure 10 shows variation of mean FBF values over time. Movement artifacts are identified by blue arrows in the top graph of Figure 10 which presents unprocessed mean FBF values. The bottom graph of Figure 10 shows the same graph corrected for artifacts and smoothed. Regression analysis shows that blood flow decreased linearly over time (coefficient of determination $\left.\mathrm{R}^{2}=0.9014\right)$ which explains the lower FBF at 79 minutes in Figure 9. No rhythmic pattern of fluctuations in mean FBF values can be observed.

\subsubsection{Subject F-Female, African-American, 68}

Figure 11 shows $\mathrm{FBF}$ of subject $\mathrm{F}$ after the relaxation period at 20 minutes (left image), and 43 minutes (right image). There is very little change in FBF between these 2 images. The time period shown in Figure 12 is about 45 minutes because the subject was disturbed by someone inadvertently entering the room at that time. Only one movement artifact can be seen in the top graph of Figure 12, which presents change in unprocessed mean FBF values over time. Bottom graph of Figure 12 shows change in mean FBF values over time corrected for that one artifact and smoothed. In her case, mean FBF fluctuated up and down for about 20 minutes (corresponding to the relaxation period) before settling down to a low stable value. This stable value after the initial relaxation period explains why the two images of Figure 11 show about the same level of FBF. No rhythmic pattern in mean FBF value can be observed after the relaxation period.
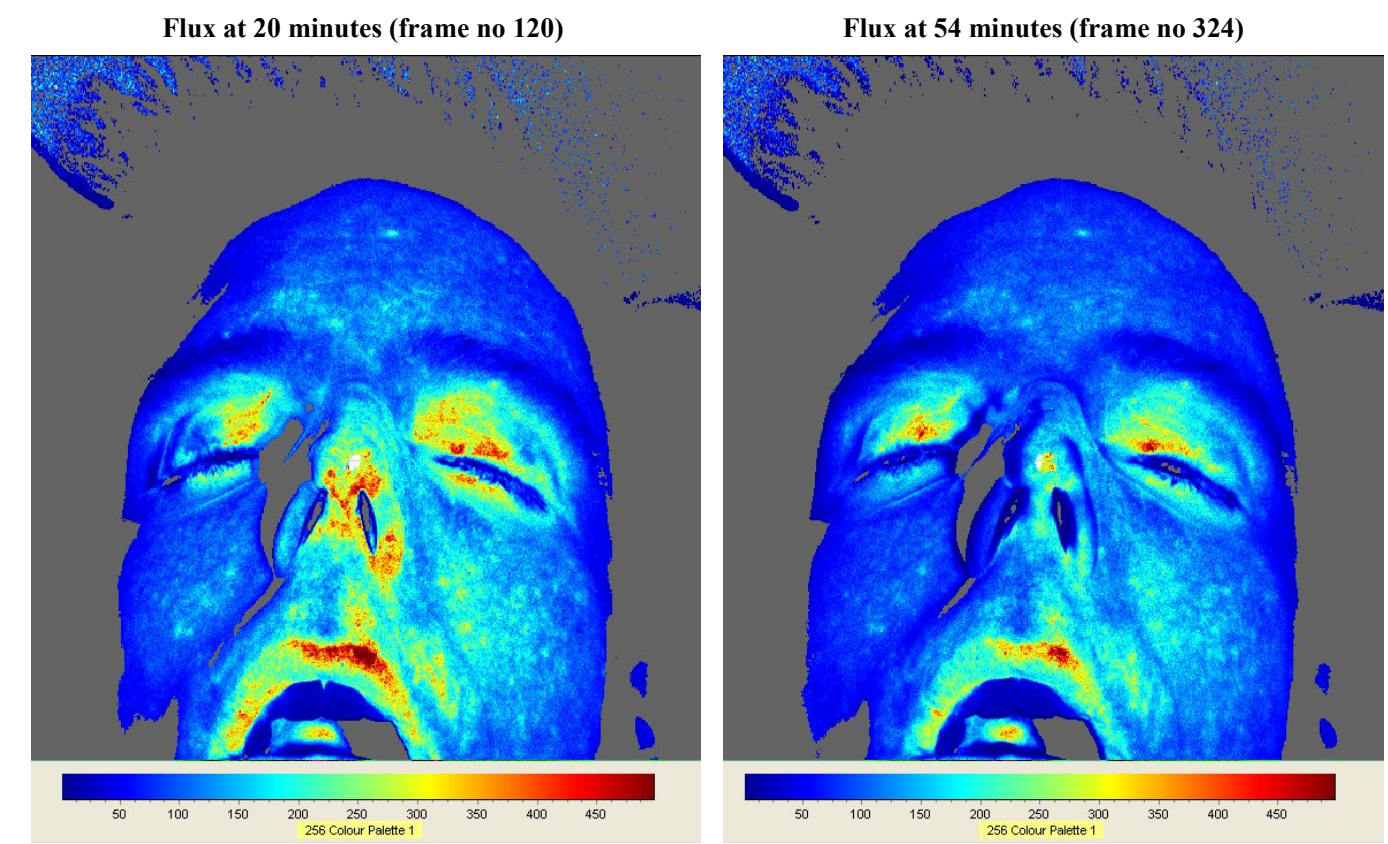

Figure 7. Subject D FBF images at 20 minutes (left image) and 54 minutes (right image). There is lower FBF in the right image compared to the left image. Descriptive statistics for the left image: Mean Flux $=127.9 ; \mathrm{SD}$ $=80.1$; Flux $\min =0$; Flux $\max =784$. Descriptive statistics for the right image: Mean Flux $=103.1 ; \mathrm{SD}=$ 63.6; Flux $\min =0$; Flux $\max =705$. 


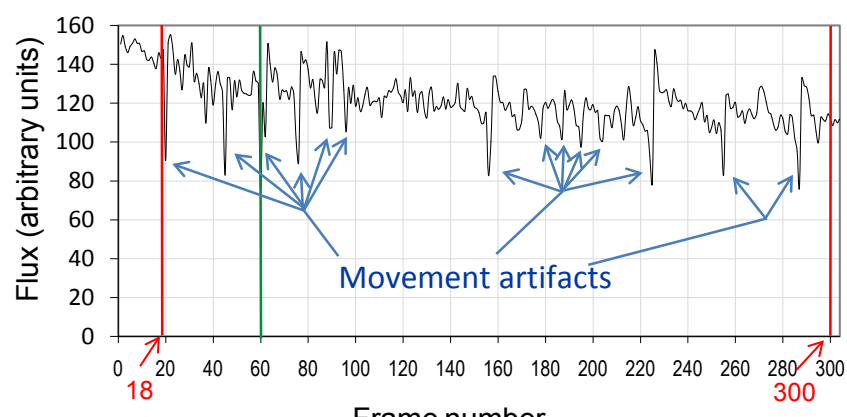

Frame number

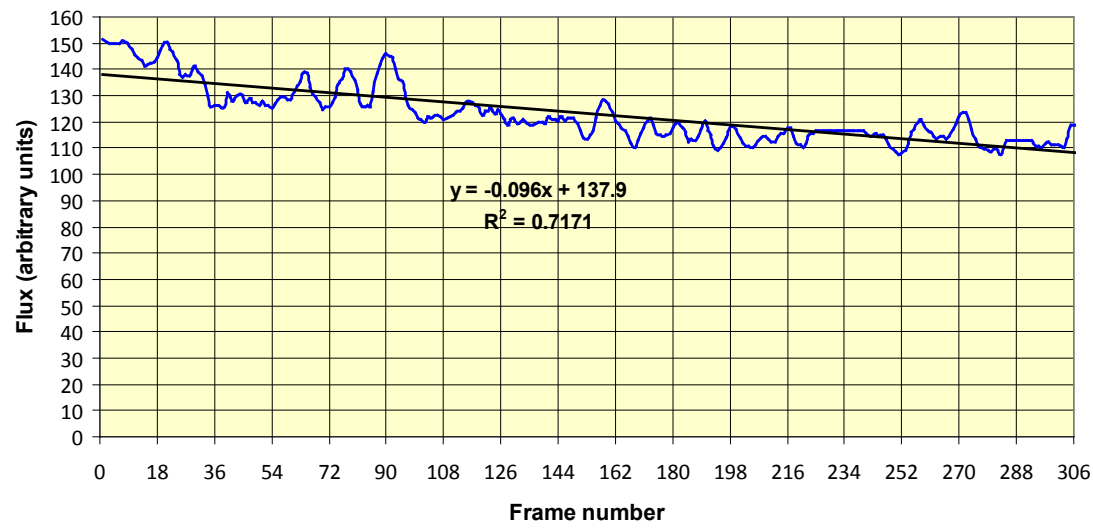

Figure 8. Top graph: Subject D unprocessed graph of mean FBF (in arbitrary units) over time. Red lines and numbers show the two frames at which the images of Figure 7 were extracted from the video. Blue arrows point to dips in flux caused by movement artifacts. The green line at 60 shows the time when the switch was flipped (no grounding occurred). Bottom graph: Subject D graph of mean FBF (in arbitrary units) over time corrected for movement artifacts and smoothed with linear regression line, equation and $\mathrm{R}^{2}$ value.

Flux at 20 minutes (frame no 120)

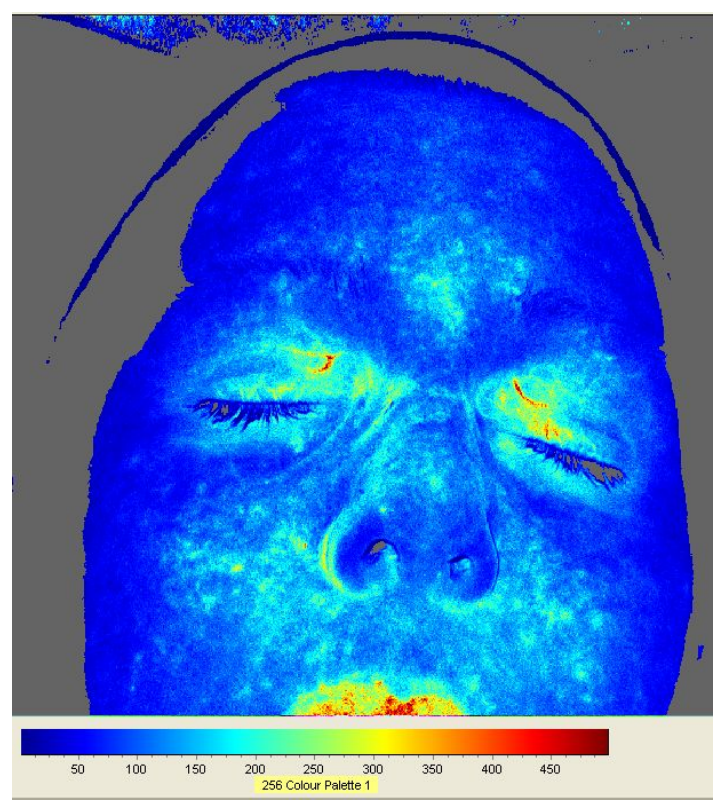

Flux at 79 minutes (frame no 474)

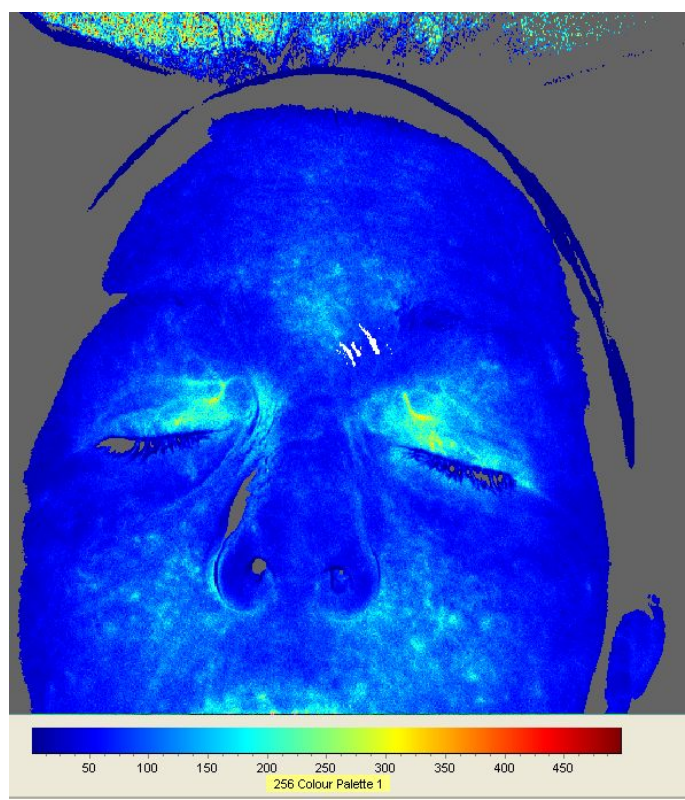

Figure 9. Subject E FBF images at 20 minutes (left image) and 79 minutes (right image). There is lower FBF in the right image compared to the left image. Descriptive statistics for the left image: Mean Flux =103.7; SD = 55.9; Flux $\min =0 ;$ Flux $\max =606$. Descriptive statistics for the right image: Mean Flux $=73.0 ; \mathrm{SD}=46.0 ;$ Flux $\min =0$; Flux $\max =730$. 

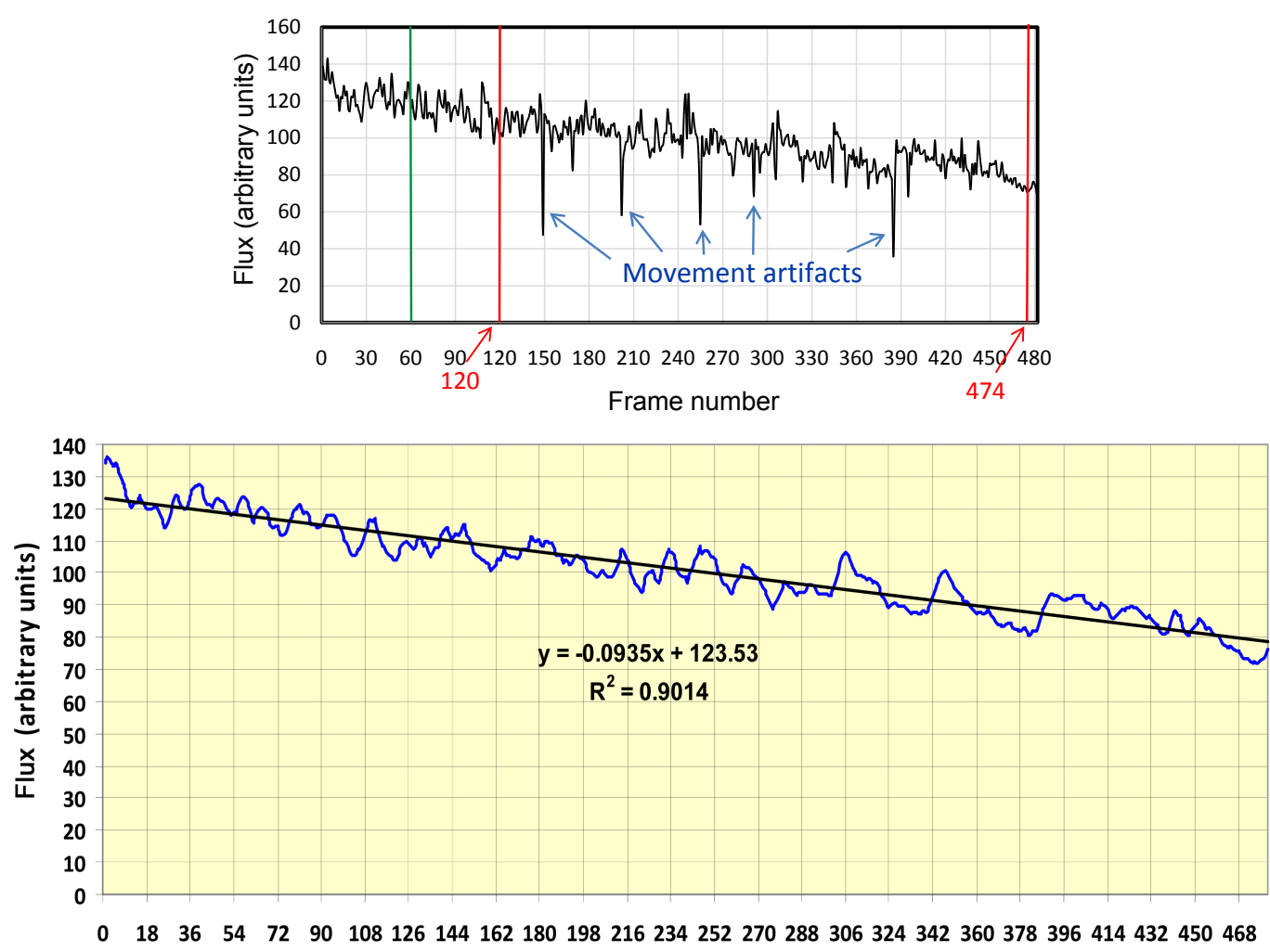

Frame number

Figure 10. Top graph: Subject E unprocessed graph of mean FBF (in arbitrary units) over time. Red lines and numbers show the time at which the two images of Figure 9 were extracted from the video. Blue arrows point to dips in flux caused by movement artifacts. The green line at 60 shows the time when the switch was flipped (no grounding occurred). Bottom graph: Subject E mean FBF graph (in arbitrary units) over time corrected for movement artifacts and smoothed with linear regression line, equation and $\mathrm{R}^{2}$ value.

Flux at 20 minutes (frame no 120)

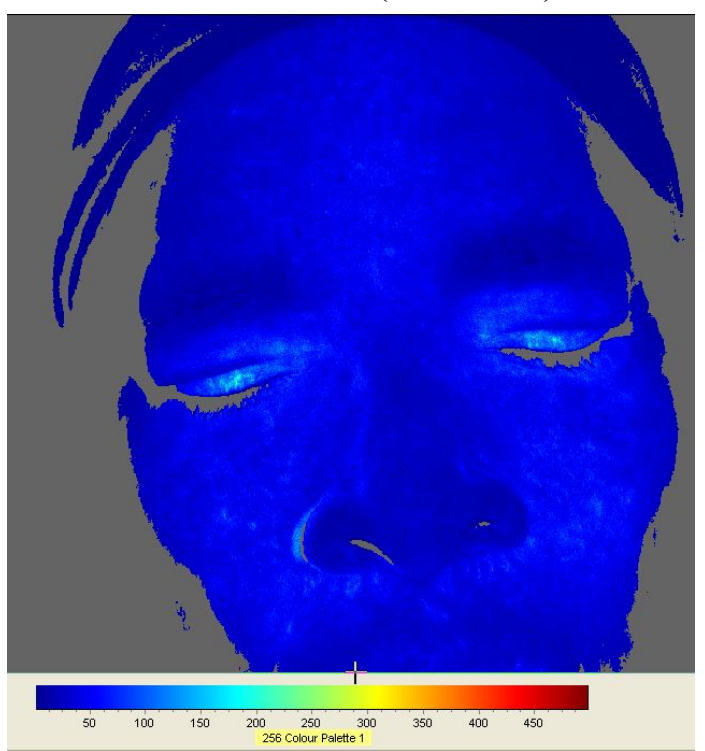

Flux at 43 minutes (frame no 258)

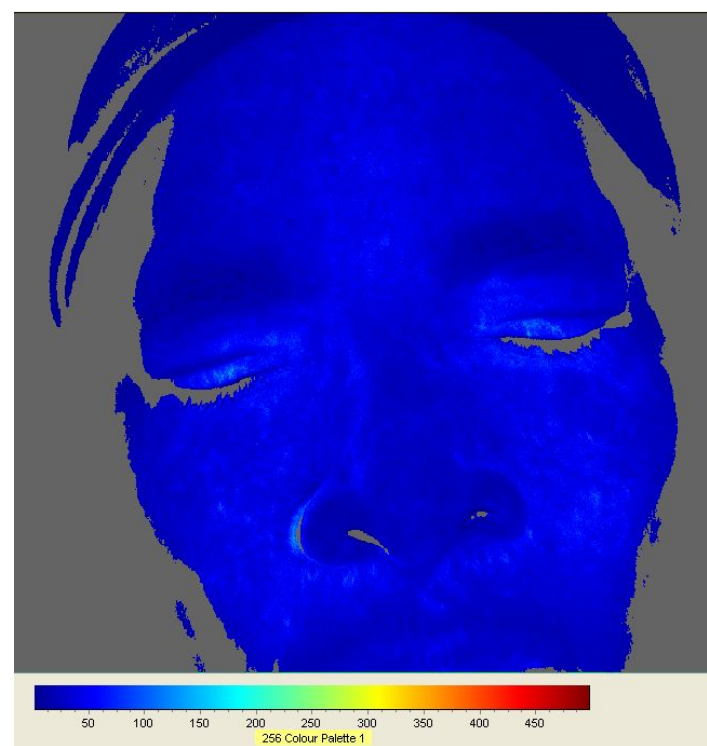

Figure 11. Subject F FBF images at 20 minutes (left image) and 43 minutes (right image). There is very little change in FBF. Descriptive statistics for the left image: Mean Flux $=33.4$; $\mathrm{SD}=19.1$; Flux $\min =0$; Flux $\max =248$. Descriptive statistics for the right image: Mean Flux $=29.4 ; \mathrm{SD}=15.8 ;$ Flux $\min =0 ;$ Flux $\max =160$. 

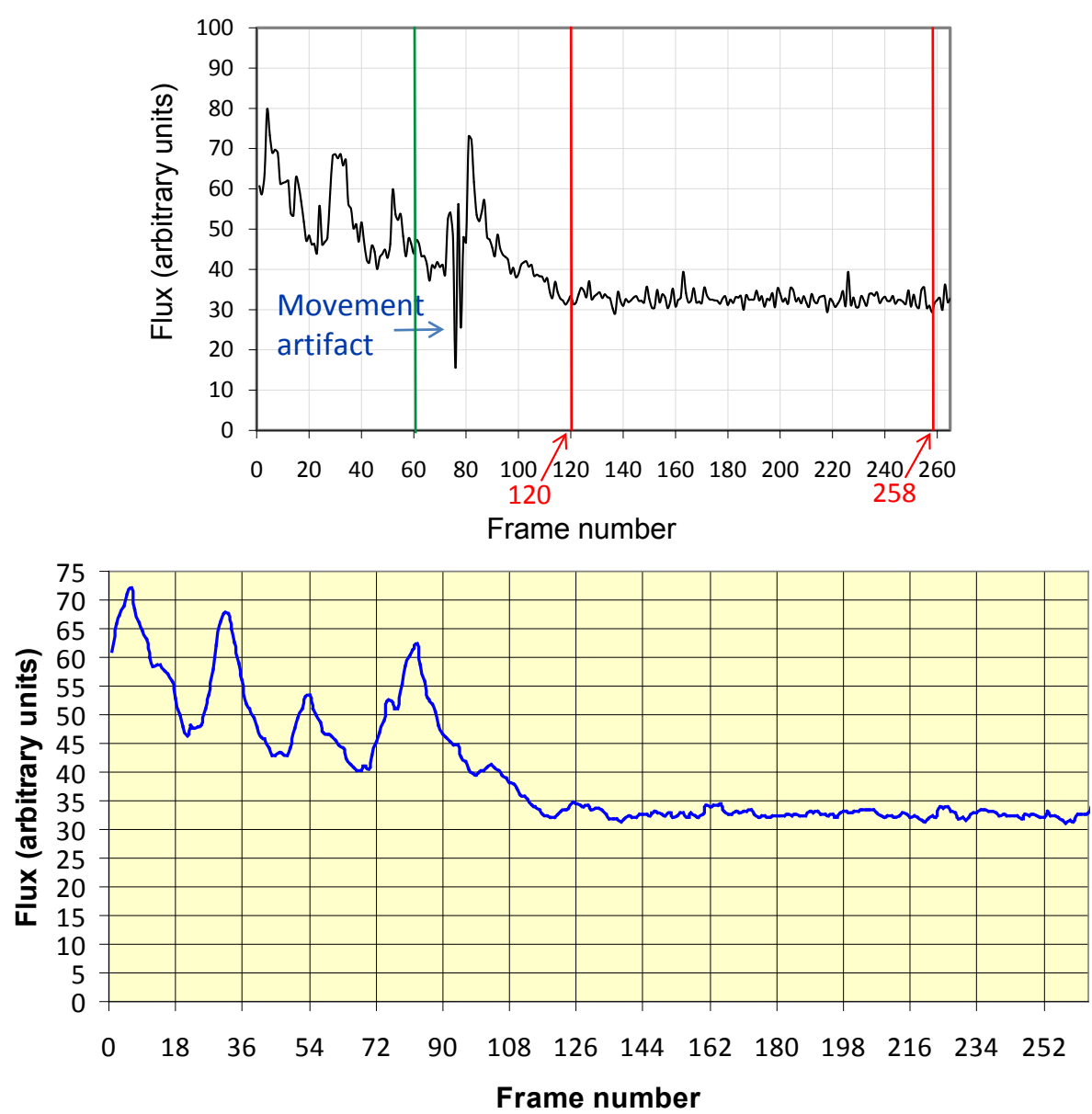

Figure 12. Top graph: Subject $\mathrm{F}$ unprocessed graph of mean FBF (in arbitrary units). Red lines and numbers show the time at which the two images of Figure 11 were extracted. The blue arrow points to a dip in flux caused by movement artifacts. The green line at 60 shows the time when the switch was flipped (no grounding occurred). Bottom graph: Subject F graph of mean FBF (in arbitrary units) corrected for movement artifacts and smoothed.

\section{Discussion}

The purpose of this study was to determine if grounding the body promotes FBF. Improved facial microcirculation is a goal of various treatments used in the beauty industry. They include massage, the use of current emitting devices (DC and AC), lasers, ultrasound emitting devices, and acupuncture [8] [9]. Improved circulation may generate enhanced nourishment of the skin through greater delivery of oxygen and nutrients, as well as better resistance to the oxidative aging process [13].

The results of the present study showed improved FBF regulation in grounded subjects only. During the experimental period of about one hour, the FBF in these subjects, as documented by the LSCI camera, was seen to fluctuate with a regular rhythm and/or increase after an initial relaxation period varying from 12 to 29 minutes. By comparison, FBF decreased steadily and/or remained constant at a low value after a relaxation period during sham-grounded sessions of similar length with no apparent rhythmicity.

There are at least four neuronal mechanisms influencing FBF. Three of them are controlled by the sympathetic or parasympathetic nervous systems while the fourth one relates to local inflammatory responses [14]. Therefore, the present results suggest that connection with the Earth supports a more efficient autonomic nervous system (ANS) regulation of FBF. The periodicity of mean FBF fluctuations appeared in approximate 4 minute increments. While the reason for this incremental length in periodicity is not known, it is interesting to note that a rhythmic pattern of contraction/relaxation was seen for the first time in muscle tension after a grounding period of 28 minutes [15]. In relation to these observations, it is also interesting to mention that the baroreflex system, a 
mechanism by which the ANS control blood pressure, operates in a frequency range that overlaps our present observations [16]-[18].

The ANS regulation of FBF dynamics brings to mind an analogy of an efficient thermostat that activates or deactivates the heating or cooling system according to temperature fluctuations within the controlled environment. For the body, grounding may contribute to the restoration of regulation by the ANS of the distribution of blood, and therefore needed oxygen and nutrients, to the various organs and systems according to their needs.

Another explanation for improved FBF produced by grounding likely relates to the zeta potential (ZP) and aggregation of red blood cells (RBCs). RBC membranes naturally carry a negative electric charge that maintains cell spacing in the bloodstream by electrostatic repulsion. The potential difference between the RBC surface and the plasma produced by these charges is called zeta potential (ZP). ZP is an indicator of blood viscosity [19][21]. Elevated blood viscosity is associated with a number of clinical conditions, including hypertension, smoking, lipid disorders, advancing age, and diabetes mellitus. Research has found, for instance, a poor ZP among diabetics, and poorer yet among diabetics with cardiovascular disease [19]. The more negative the RBC surface charge is, the greater the repelling force between RBCs implying that the viscosity of the blood is lower which results in an improved blood flow [19]-[21]. In a previous study, grounding improved ZP and reduced RBC aggregation. Among the ten participants, the absolute value of the average ZP increased by a factor of 2.70-almost three-fold [21].

A third explanation for improved FBF is accumulated evidence that grounding improves overall physiology. If the body is healthier, it follows that the skin should be in better condition [8]. In support of this assertion, grounding has been shown to improve recovery from injury [22] [23], thyroid function and basic metabolism, calcium metabolism, glucose utilization by cells, the immune response [24], and oxygen consumption [25]. Some researchers consider that grounding may even be the primary factor regulating the endocrine and nervous systems [24].

It is instructive to raise the issue of stress. Numerous studies indicate that stress-induced sleep deficit can dramatically impair skin function and integrity [26]-[30]. Chronic insomnia, experienced by as much as a third of adults, can create damage to skin tissues ranging from premature aging [31] [32] to disorders like eczema, psoriasis, and dermatitis [33]-[36]. Previous grounding studies have produced results in which grounded participants subjectively reported better sleep and show lower stress levels [15] [25] [37]-[39].

According to the American Academy of Dermatology, stress can affect the skin in many ways [40]. Stress causes abnormalities in the level and oscillation of the central stress hormone cortisol that regulates a wide range of stress responses. Such disruption can trigger multiple neuroinflammatory conditions manifested in the skin, such as psoriasis, atopic dermatitis, acne, contact dermatitis alopecia areata, itch or pruritus, and erythema [41] [42]. Along with better sleep, grounding at night has been demonstrated to bring aberrant cortisol oscillations more in line with the natural cortisol pattern [37]. It should also be noted that grounding appears to promote balance in the sympathetic-parasympathetic function of the ANS, and thus exerts another stress reduction effect [15] [25] [39].

Through various measurements, grounding has also been documented to reduce inflammation [22] [43]. One mechanism of inflammation reduction is hypothesized to be the neutralization of oxidative free radical activity by added free electrons from contact with the Earth [4] [5]. Oxidative stress plays a central role in initiating and driving events that cause skin aging at the cellular level [44]. Oxidative stress breaks down protein (collagen), alters cellular renewal cycles, damages DNA, and promotes collagen glycation, cross-linking of proteins, and the release of pro-inflammatory mediators (cytokines), which trigger the generation of inflammatory skin diseases. It is also established that free radicals participate in the pathogenesis of allergic reactions in the skin [44]-[50]. The grounding effect may also be protective and/or therapeutic against UV radiation that produces oxidative stress in the cellular environment of the skin. Chronic free radical assault leads to aging skin, subverting the structural framework of the skin, and giving rise to wrinkles and sagging skin [46].

Another mechanism of inflammation reduction is the inflammatory reflex. Discovered about 15 years ago, this neural reflex mechanism controls inflammation and innate immune responses during tissue injury and pathogen invasion [51] [52]. A major constituent of the inflammatory reflex is the vagus nerve. Since grounding stimulates the parasympathetic branch of the ANS, it is reasonable to theorize that vagus nerve-mediated cholinergic signaling is also stimulated resulting in a decrease in inflammation.

Along with previous studies, the results of this study indicates that extended periods of grounding could be expected to produce further changes and benefits to facial skin. There's a saying that beauty comes from within. 
It may also be appropriate to say that the beauty within comes from the ground.

\section{Conclusion}

The very Earth we live on possesses a form of easily accessible and beneficial natural electric energy that has been found to positively influence human physiology in various ways [6]. Previous studies have indicated improved cardiovascular and rheological (blood viscosity) dynamics, including autonomic nervous system regulation. In this study, the Laser Speckle Contrast Imaging camera further supports those findings by documenting a clear improvement in autonomic nervous system regulation of facial blood flow in grounded subjects but not in sham-grounded subjects. The results demonstrate, for the first time, that even one-hour contact with the Earth restores blood flow regulation to the face that may enhance skin tissue repair, health and vitality, and optimize facial appearance, which may also have broad implications for overall cardiovascular function and health. Further studies, using larger comparison groups, longer monitoring times and more measuring methods, are warranted in order to confirm the novel influence of the Earth as a protector of skin health and appearance.

\section{Acknowledgements}

The author wishes to thank Earthing.com for providing the grounding equipment, Linda Hayes, C.C.T. and Theresa Williams, C.C.T., from Total Thermal Imaging, for recruiting study participants, collecting data and conducting all imaging activities, and Martin Zucker for reviewing the manuscript, for writing assistance, and for making useful suggestions. The study was funded by Earth FX, Inc.

\section{Conflict of Interests}

The author is an independent contractor for Earth FX, Inc. and owns a very small number of shares in the company.

\section{References}

[1] Williams, E. and Heckman, S. (1993) The Local Diurnal Variation of Cloud Electrification and the Global Diurnal Variation of Negative Charge on the Earth. Journal of Geophysical Research, 98, 5221-5234. http://dx.doi.org/10.1029/92JD02642

[2] Anisimov, S., Mareev, E. and Bakastov, S. (1999) On the Generation and Evolution of Aeroelectric Structures in the Surface Layer. Journal of Geophysical Research, 104, 14359-14367. http://dx.doi.org/10.1029/1999JD900117

[3] Applewhite, R. (2005) The Effectiveness of a Conductive Patch and a Conductive Bed Pad in Reducing Induced Human Body Voltage via the Application of Earth Ground. European Biology and Bioelectromagnetics, 1, 23-40. http://www.earthingoz.com.au/pdf/Applewhite_earthing_body_voltage_2005.pdf

[4] Oschman, J.L. (2009) Charge Transfer in the Living Matrix. Journal of Bodywork and Movement Therapies, 13, $215-$ 228. http://dx.doi.org/10.1016/j.jbmt.2008.06.005

[5] Oschman, J.L. (2007) Can Electrons Act as Antioxidants? A Review and Commentary. Journal of Alternative and Complementary Medicine, 13, 955-967. http://online.liebertpub.com/doi/pdfplus/10.1089/acm.2007.7048 http://dx.doi.org/10.1089/acm.2007.7048

[6] Chevalier, G., Sinatra, S.T., Oschman, J.L., Sokal, K. and Sokal, P. (2012) Earthing: Health Implications of Reconnecting the Human Body to the Earth's Surface Electrons. Journal of Environmental and Public Health, 2012, Article ID 291541. http://dx.doi.org/10.1155/2012/291541

[7] Med-Health.net (2014) How to Tighten Face Skin. http://www.med-health.net/How-To-Tighten-Face-Skin.html

[8] Herman, J., Rost-Roszkowska, M. and Skotnicka-Graca, U. (2013) Skin Care during the Menopause Period: Noninvasive Procedures of Beauty Studies. Postepy Dermatologii i Alergologi, 30, 388-395. http://www.ncbi.nlm.nih.gov/pmc/articles/PMC3907896/

[9] Avci, P., Gupta, A., Sadasivam, M., Vecchio, D., Pam, Z., Pam, N. and Hamblin, M.R. (2013) Low-Level Laser (Light) Therapy (LLLT) in Skin: Stimulating, Healing, Restoring. Seminars in Cutaneous Medicine and Surgery, 32, 41-52. http://www.ncbi.nlm.nih.gov/pmc/articles/PMC4126803/

[10] Briers, J.D. and Webster, S. (1996) Laser Speckle Contrast Analysis (LASCA): A Nonscanning, Full-Field Technique for Monitoring Capillary Blood Flow. Journal of Biomedical Optics, 1, 174-179. http://dx.doi.org/10.1117/12.231359

[11] Eriksson, S., Nilsson, J., Lindell, G. and Sturesson, C. (2014) Laser Speckle Contrast Imaging for Intraoperative Assessment of Liver Microcirculation: A Clinical Pilot Study. Medical Devices: Evidence and Research, 7, $257-261$. 
http://dx.doi.org/10.2147/MDER.S63393

[12] Moor, F.L.P.I. (2012) User Manual, Issue 8.

[13] Schwartz, S.R. and Park, J. (2012) Ingestion of BioCell Collagen ${ }^{\circledR}$, a Novel Hydrolyzed Chicken Sternal Cartilage Extract; Enhanced Blood Microcirculation and Reduced Facial Aging Signs. Clinical Interventions in Aging, 7, 267-273. http://dx.doi.org/10.2147/CIA.S32836

[14] Drummond, P.D. (1994) Sweating and Vascular Responses in the Face: Normal Regulation and Dysfunction in Migraine, Cluster Headache and Harlequin Syndrome. Clinical Autonomic Research, 4, 273-285. http://dx.doi.org/10.1007/BF01827433

[15] Chevalier, G., Mori, K. and Oschman, J.L. (2006) The Effect of Earthing (Grounding) on Human Physiology. European Biology and Bioelectromagnetics, 2, 600-621. http://www.barefoothealth.com/science/physiology_study.pdf

[16] Task Force of the European Society of Cardiology and the North American Society of Pacing and Electrophysiology (1996) Heart Rate Variability: Standards of Measurement, Physiological Interpretation and Clinical Use. Circulation, 93, 1043-1065. http://circ.ahajournals.org/content/93/5/1043.long http://dx.doi.org/10.1161/01.CIR.93.5.1043

[17] Goldstein, D.S., Bentho, O., Park, M.Y. and Sharabi, Y. (2011) Low-Frequency Power of Heart Rate Variability Is Not a Measure of Cardiac Sympathetic Tone but May Be a Measure of Modulation of Cardiac Autonomic Outflows by Baroreflexes. Experimental Physiology, 96, 1255-1261. http://ep.physoc.org/content/96/12/1255.long http://dx.doi.org/10.1113/expphysiol.2010.056259

[18] Bonyhay, I., Risk, M. and Freeman, R. (2013) High-Pass Filter Characteristics of the Baroreflex: A Comparison of Frequency Domain and Pharmacological Methods. PLOS ONE, 8, e79513. http://dx.doi.org/10.1371/journal.pone.0079513

[19] Adak, S., Chowdhury, S. and Bhattacharyya, M. (2008) Dynamic and Electrokinetic Behavior of Erythrocyte Membrane in Diabetes Mellitus and Diabetic Cardiovascular Disease. Biochimica et Biophysica Acta, 1780, 108-115. http://dx.doi.org/10.1016/j.bbagen.2007.10.013

[20] Fontes, A., Fernandes, H.P., de Thomaz, A.A., Barjas-Castro, M.L. and Cesar, C.L. (2008) Measuring Electrical and Mechanical Properties of Red Blood Cells with Double Optical Tweezers. Journal of Biomedical Optics, 13, Article ID: 014001. http://dx.doi.org/10.1117/1.2870108

[21] Chevalier, G., Sinatra, S.T., Oschman, J.L. and Delany, R.M. (2013) Earthing (Grounding) the Human Body Reduces Blood Viscosity: A Major Factor in Cardiovascular Disease. Journal of Alternative and Complementary Medicine, 19, 102-110. http://dx.doi.org/10.1089/acm.2011.0820

[22] Brown, R., Chevalier, G. and Hill, M. (2010) Pilot Study on the Effect of Grounding on Delayed-Onset Muscle Soreness. Journal of Alternative and Complementary Medicine, 16, 265-273. http://dx.doi.org/10.1089/acm.2009.0399

[23] Ober, C., Sinatra, S.T. and Zucker, M. (2010) Earthing: The Most Important Health Discovery Ever? Basic Health Publications, Laguna Beach, 193-205.

[24] Sokal, K. and Sokal, P. (2011) Earthing the Human Body Influences Physiologic Processes. Journal of Alternative and Complementary Medicine, 17, 301-308. http://dx.doi.org/10.1089/acm.2010.0687

[25] Chevalier, G. (2010) Changes in Pulse Rate, Respiratory Rate, Blood Oxygenation, Perfusion Index, Skin Conductance and Their Variability Induced during and after Grounding Human Subjects for 40 Minutes. Journal of Alternative and Complementary Medicine, 16, 81-87. http://dx.doi.org/10.1089/acm.2009.0278

[26] Kahan, V., Andersen, M.L., Tomimori, J. and Tufikm, S. (2010) Can Poor Sleep Affect Skin Integrity? Medical Hypotheses, 75, 535-537. http://dx.doi.org/10.1016/j.mehy.2010.07.018

[27] Ghadially, R., Brown, B.E., Sequeira-Martin, S.M., Feingold, K.R. and Elias, P. (1995) The Aged Epidermal Permeability Barrier. Structural, Functional and Lipid Biochemical Abnormalities in Humans and a Senescent Murine Model. The Journal of Clinical Investigation, 95, 2281-2290. http://dx.doi.org/10.1172/JCI117919

[28] Gupta, M.A. and Gupta, A.K. (1996) Psychodermatology: An Update. Journal of the American Academy of Dermatology, 34, 1030-1046. http://dx.doi.org/10.1016/S0190-9622(96)90284-4

[29] Tausk, F.A. and Nousari, H. (2001) Stress and the Skin. Archives of Dermatology, 137, 78-82. http://dx.doi.org/10.1001/archderm.137.1.78

[30] Grice, K.A. (1980) Transepidermal Water Loss in Pathologic Skin. In: Jarrett, A., Ed., The Physiology and Pathophysiology of the Skin, Academic Press, London, 2147-2155.

[31] Rööst, M. and Nilsson, P. (2002) Sleep Disorders-A Public Health Problem. Potential Risk Factor in the Development of Type 2 Diabetes, Hypertension, Dyslipidemia and Premature Aging. Läkartidningen, 99, 154-157.

[32] Edwards, B.A., O’Driscoll, D.M., Ali, A., Jordan, A.S., Trinder, J. and Malhotra, A. (2010) Aging and Sleep: Physiology and Pathophysiology. Seminars in Respiratory and Critical Care Medicine, 31, 618-633. 
http://dx.doi.org/10.1055/s-0030-1265902

[33] Altemus, M., Rao, B., Dhabhar, F.S., Ding, W. and Granstein, R.D. (2001) Stress-Induced Changes in Skin Barrier Function in Healthy Women. The Journal of Investigative Dermatology, 7, 309-317. http://dx.doi.org/10.1046/j.1523-1747.2001.01373.x

[34] Kobayashi, S., Hayashi, K., Koyama, S., Tsubaki, H., Itano, T., Momomura, M., Koyama, T. and Yanagawa, Y. (2010) Actigraphy for the Assessment of Sleep Quality in Pediatric Atopic Dermatitis Patients. Arerugi, 59, 706-715.

[35] Hanifin, J.M. and Reed, M.L., Eczema Prevalence and Impact Working Group (2007) A Population-Based Survey of Eczema Prevalence in the United States. Dermatitis, 18, 82-91. http://dx.doi.org/10.2310/6620.2007.06034

[36] Choi, E.H., Brown, B.E., Crumrine, D., Chang, S., Man, M.-Q., Elias, P.M. and Feingold, K.R. (2005) Mechanisms by Which Psychologic Stress Alters Cutaneous Permeability Barrier Homeostasis and Stratum Corneum Integrity. Journal of Investigative Dermatology, 124, 587-595. http://dx.doi.org/10.1111/j.0022-202X.2005.23589.x

[37] Ghaly, M. and Teplitz, D. (2004) The Biologic Effects of Grounding the Human Body during Sleep as Measured by Cortisol Levels and Subjective Reporting of Sleep, Pain and Stress. Journal of Alternative and Complementary Medicine, 10, 767-776. http://74.63.154.231/here/wp-content/uploads/2013/06/Ghaly Teplitz cortisol study 2004.pdf http://dx.doi.org/10.1089/acm.2004.10.767

[38] Ober, C. (2000) Grounding the Human Body to Neutralize Bioelectrical Stress from Static Electricity and EMFs. ESD Journal. www.esdjournal.com/articles/cober/ground.htm

[39] Chevalier, G. and Sinatra, S.T. (2011) Emotional Stress, Heart Rate Variability, Grounding and Improved Autonomic Tone: Clinical Applications. Integrative Medicine: A Clinician's Journal, 10, 16-21. http://imjournal.com/pdfarticles/IMCJ10 3 p16 24chevalier.pdf

[40] American Academy of Dermatology (2014) Stress and Skin. http://www.aad.org/media-resources/stats-and-facts/prevention-and-care/stress-and-skin

[41] Senra, M.S. and Wollenberg, A. (2014) Psychodermatological Aspects of Atopic Dermatitis. British Journal of Dermatology, 170, 38-43. http://dx.doi.org/10.1111/bjd.13084

[42] Chen, Y. and Lyga, J. (2014) Brain-Skin Connection: Stress, Inflammation and Skin Aging. Inflammation \& Allergy Drug Targets, 13, 177-190. http://www.ncbi.nlm.nih.gov/pmc/articles/PMC4082169/ http://dx.doi.org/10.2174/1871528113666140522104422

[43] Oschman, J.L., Chevalier, G. and Brown, D. (2014) The Effects of Grounding (Earthing) on Inflammation, the Immune Response, Wound Healing and Prevention and Treatment of Chronic Inflammatory and Auto-Immune Diseases. Journal of Inflammation Research, in Press.

[44] Masaki, H. (2010) Role of Antioxidants in the Skin: Anti-Aging Effects. Journal of Dermatological Science, 58, 85-90. http://dx.doi.org/10.1016/j.jdermsci.2010.03.003

[45] Burke, K.E. and Wei, H. (2009) Synergistic Damage by UVA Radiation and Pollutants. Toxicology and Industrial Health, 25, 219-224. http://tih.sagepub.com/content/25/4-5/219

[46] Fisher, G.J., Quan, T., Purohit, T., Shao, Y., Cho, M.K., He, T., Varani, J., Kamg, S. and Voorhees, J. (2009) Collagen Fragmentation Promotes Oxidative Stress and Elevates Matrix Metalloproteinase-1 in Fibroblasts in Aged Human Skin. The American Journal of Pathology, 174, 101-114. http://dx.doi.org/10.2353/ajpath.2009.080599

[47] Pascucci, B., D'Errico, M., Parlanti, E., Giovannini, S. and Dogliotti, E. (2011) Role of Nucleotide Excision Repair Proteins in Oxidative DNA Damage Repair: An Updating. Biochemistry (Moscow), 76, 4-15. http://dx.doi.org/10.1134/S0006297911010032

[48] Röck, K., Grandoch, M., Majora, M., Krutmann, J. and Fisher, J.W. (2011) Collagen Fragments Inhibit Hyaluronan Synthesis in Skin Fibroblasts in Response to Ultraviolet B (UVB): New Insights into Mechanisms of Matrix Remodeling. The Journal of Biological Chemistry, 286, 18268-18276. http://dx.doi.org/10.1074/jbc.M110.201665 Supplemental Material: http://www.jbc.org/content/suppl/2011/03/17/M110.201665.DC1.html

[49] Daniel, S., Reto, M. and Fred, Z. (2002) Collagen Glication and Skin Aging. Cosmetics and Toiletries Manufacture Worldwide. https://www.mibellebiochemistry.com/pdfs/Collagen glycation and skin aging - CT 2002.pdf

[50] Miwa, S., Beckman, K.B. and Muller, F., Eds. (2008) Oxidative Stress in Aging: From Model Systems to Human Diseases. Humana Press, New York.

[51] Borovikova, L.V., Ivanova, S., Zhang, M., Yang, H., Botchkina, G.I., Watkins, L.R., Wang, H., Abumrad, N., Eaton, J.W. and Tracey, K.J. (2000) Vagus Nerve Stimulation Attenuates the Systemic Inflammatory Response to Endotoxin. Nature, 405, 458-462. http://dx.doi.org/10.1038/35013070

[52] Pavlov, V.A. and Tracey, K.J. (2012) The Vagus Nerve and the Inflammatory Reflex-Linking Immunity and Metabolism. Nature Reviews Endocrinology, 8, 743-754. http://dx.doi.org/10.1038/nrendo.2012.189 
Scientific Research Publishing (SCIRP) is one of the largest Open Access journal publishers. It is currently publishing more than 200 open access, online, peer-reviewed journals covering a wide range of academic disciplines. SCIRP serves the worldwide academic communities and contributes to the progress and application of science with its publication.

Other selected journals from SCIRP are listed as below. Submit your manuscript to us via either submit@scirp.org or Online Submission Portal.
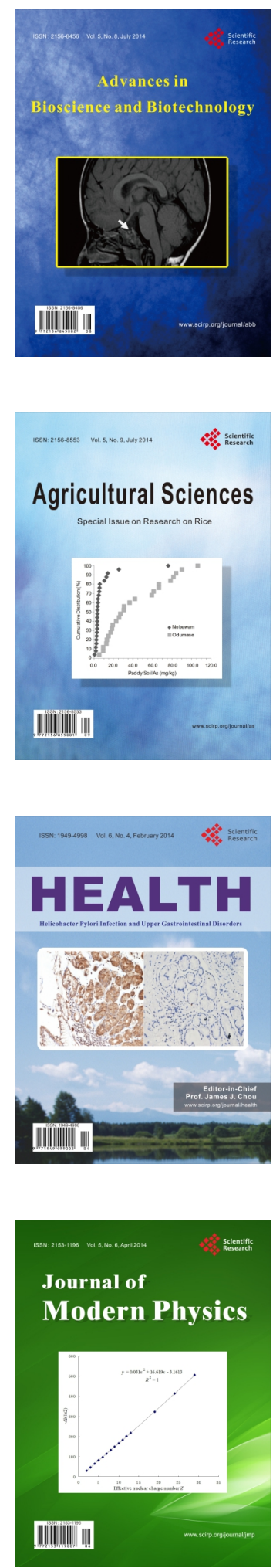
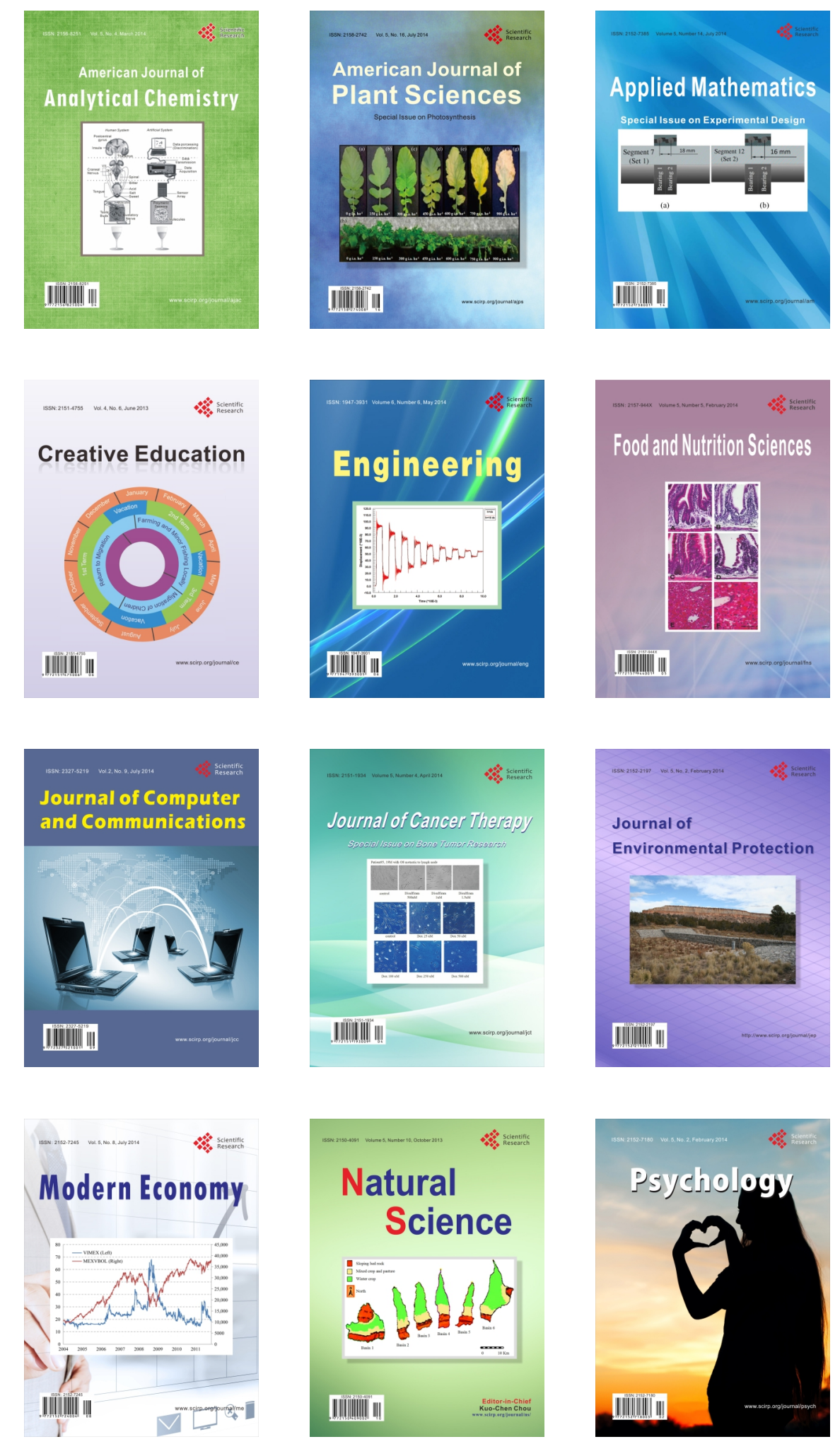